https://doi.org/10.15407/ujpe64.4.354

L.O. MAZUR ${ }^{1}$ L.A. BULAVIN,${ }^{1}$ N.V. VYGORNITSKII, ${ }^{2}$ N.I. LEBOVKA ${ }^{2,1}$

1 Taras Shevchenko National University, Faculty of Physics

(2, Acad. Glushkov Ave., Kyiv 03127, Ukraine; e-mail: liliamazur@outlook.com)

${ }^{2}$ F.D. Ovcharenko Institute of Biocolloidal Chemistry, NAS of Ukraine

(42, Vernadsky Ave., 03142 Kyiv, Ukraine)

\title{
EFFECTS OF BROWNIAN MOTIONS \\ ON ELECTRICAL CONDUCTIVITY AND OPTICAL TRANSPARENCY OF TWO-DIMENSIONAL FILMS FILLED BY NEEDLE-LIKE PARTICLES
}

\section{Introduction}

Conductive films with high optical transparency can be of great use in solar panels, lasers, photo and video cameras, touch screens, pressure and deformation sensors, and flexible optical devices [1-3]. Metallic oxides (e.g., indium tin oxide) are traditionally used to make transparent and conductive films. However, such materials are fragile, so they can degrade with the appearance of microcracks and have a high cost, which limits their use [4,5]. Recently, for the manufacture of conductive films with high transparency, the materials filled with conductive nanoparticles $[6,7]$, in particular, those based on the plates of graphene $[8,9]$ or carbon nanotubes [10], began to be used. In this case, the main problem associated with the use of carbon nanotubes is their high tendency to the aggregation and the presence of aging processes caused by the Brownian motion of particles. This can significantly affect the behavior of the conductivity and transparency of films containing carbon nanotubes. In order to avoid the aggregation and improve

\section{(C) L.O. MAZUR, L.A. BULAVIN, N.V. VYGORNITSKII,} N.I. LEBOVKA, 2019 the dispersion of nanotubes, it is possible to use surfactants $[11,12]$, supplements of nanoparticles that improve the dispersion (e.g., laponite [13-15]), and chemical functionalization of the surface of nanotubes $[16,17]$. These methods allow one to regulate the interaction between nanotubes and influence the processes of time evolution of the properties of systems.

Recently, computer research methods have been used to predict the electrophysical properties of thin films filled with anisotropic or needle-like particles. The analysis included discrete (lattice) [18] and continuous models [19, 20], disordered and partially ordered systems, effects of polydispersity of a needle length [21], as well as effects associated with the presence of defects [18]. The anisotropy of the conductivity in monolayers $[19,20]$ and the effects of self-organization/aging that arise in the presence of the Brownian diffusion [22-24] or in the presence of a vertical drying have been studied (see, in particular, [25]). The analysis of the behavior of the optical density in systems filled with ordered particles of an anisotropic form (disks or cylinders) [26] was also carried out. The studies included the consideration of only the systems, in which particles do not interact,

ISSN 2071-0194. Ukr. J. Phys. 2019. Vol. 64, No. 4 
and may [20] or may not [19] intersect. The detection of the effect of the nature of anisotropic interactions between particles on the aging processes of films and changes in their physical characteristics, in particular, the electrical conductivity and optical transmission, is of significant interest.

Therefore, the purpose of this work was to study the behavior of the electrical conductivity and optical density of two-dimensional films filled with needlelike conductive particles with anisotropic interaction between them. The aging processes as a result of the Brownian diffusion was analyzed by the MonteCarlo method with changing the parameters of an anisotropic interaction.

\section{Computer Model and Calculation Method}

The particles were shaped of needles and were represented by linear segments of unit length $(l=1)$ and zero thickness $(d=0)$, that is, they had an infinite aspect ratio of $k=l / d=\infty$. At the initial moment, the needles were planted successively (one by one) onto a plane of $L \times L$ using the RSA algorithm, in which the intersection with previously placed particles was forbidden [27]. During the precipitation of needles, their coordinates and orientations were randomly chosen, and the periodic boundary conditions were used. The numerical concentration of needles was determined as $n=N / L^{2}$, where $N$ is the total number of particles on the plane.

After the formation of an initial configuration, the further evolution of the film structure (i.e., its aging) was modeled taking the Brownian diffusion into account and using the Monte-Carlo method. The Brownian motion of a randomly chosen needle was determined by attempts to turn at the angle of $\Delta \theta$ and to displace it to a distance of $\Delta l$, by considering the next boundary ratio between their mean square values

$\lim _{k \rightarrow \infty}\left\langle\Delta \theta^{2}\right\rangle /\left\langle\Delta l^{2}\right\rangle=D_{r} / 2 D_{t}=4 / l^{2}$,

where

$D_{t}=\frac{3 k_{\mathrm{B}} T\left[\ln k+\gamma_{t}\right]}{8 \pi \eta l}, \quad D_{r}=\frac{3 k_{\mathrm{B}} T\left[\ln k+\gamma_{r}\right]}{\pi \eta l^{3}}$

are the diffusion coefficients of the translational, $D_{t}$, and rotational, $D_{r}$, motions [28], $k_{\mathrm{B}} T$ is the thermal energy, $\eta$ is the viscosity of the medium, and $\gamma_{t} \approx$ $\approx 0.219$ and $\gamma_{r} \approx-0.447$ are correction factors that are related to the hydrodynamic effects of the flow around the ends of a needle-like particle [29-31].

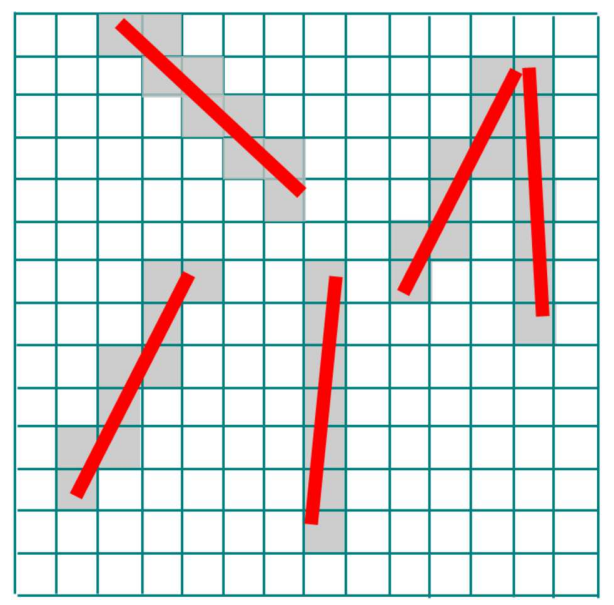

Fig. 1. The subtraction used to estimate the electrical conductivity $\sigma$ and the optical transmission $T_{r}$. The needles cover some cells (marked with dark color), and these cells have an effective electrical conductivity of $\sigma_{p}$. Unfilled (white) cells have an effective electrical conductivity of $\sigma_{m} \ll \sigma_{p}$

In view of the interaction effects between particles, the probability of their displacement or rotation is calculated as $W=\exp (-\Delta u)$, where $\Delta u$ is a change in the energy of the system in units of $k_{\mathrm{B}} T$. It is assumed that the needles interact only when the distance between them $r$ does not exceed a certain critical length $R_{c}$, and the angular dependence of the interaction potential is described by the function

$u=u_{0} \cos ^{2} \phi$

where $\phi$ is the angle between the needles, and $u_{0}$ is the energy factor. In particular, $u_{0}<0$ corresponds to the attraction between the particles, which is maximized, when the needles are parallelized.

One MC time step corresponds to $N$ attempts to move and rotate. The total simulation time was $10^{6}$ MC steps.

To calculate the electrical conductivity and optical transparency, an auxiliary mesh of $m \times m$ cells was used (see Fig. 1). The use of this auxiliary mesh actually corresponds to replacing the needle-like particle with a particle with finite aspect ratio $a$, which should be evaluated as $a=m / L$. The case of $m \rightarrow \infty$ corresponds to the needle-like particle.

To calculate the electrical conductivity, the empty cells of the mesh (which did not have a section with a needle) had the effective electrical conductivity $\sigma_{m}=1$ (the electrical conductivity of the medium in 

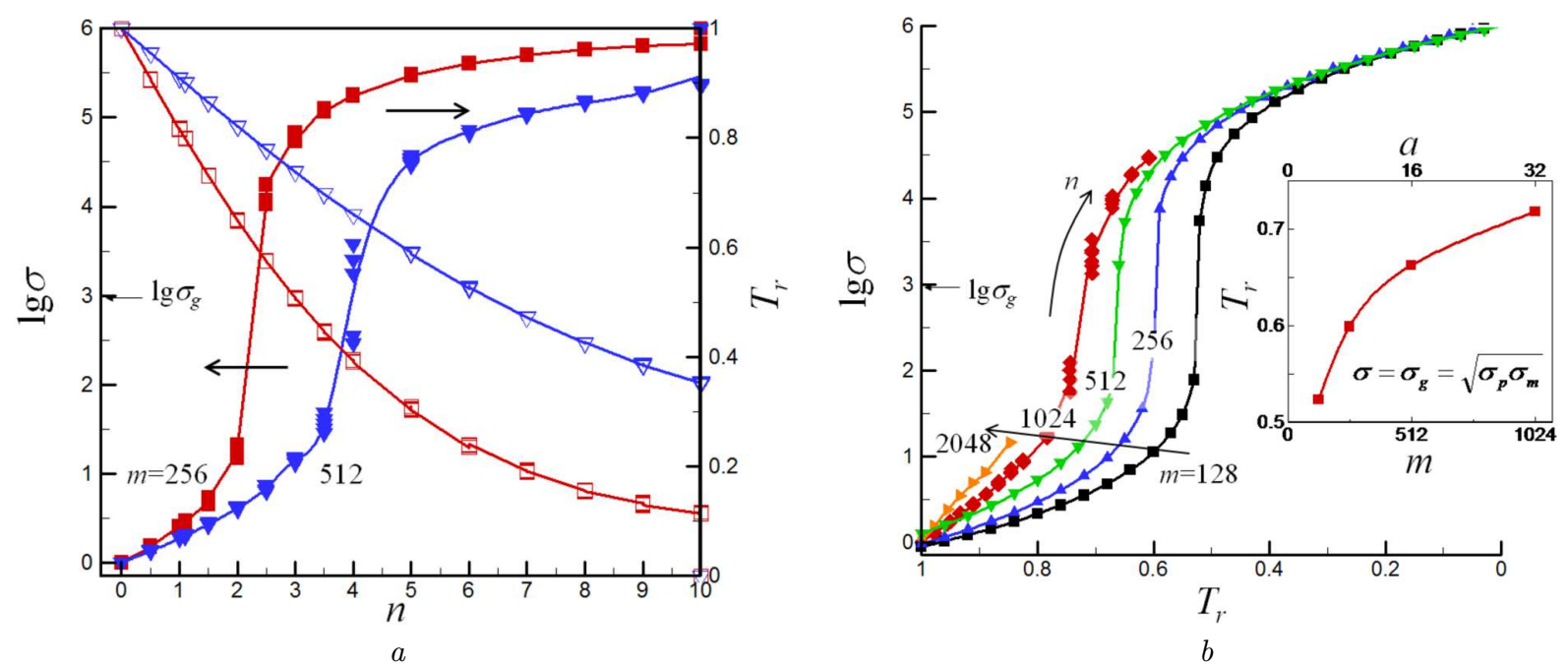

Fig. 2. Dependences of the electrical conductivity $\sigma$ and the optical transmission $T_{r}$ on the numerical concentration of needles $n$ for $m=256 a n d 512(a)$, and the dependence of the electrical conductivity $\sigma$ on the optical transmission $T_{r}$ for various $m$ (b). The data correspond to the initial time moment immediately after the formation of the system, using the RSA deposition mechanism

arbitrary units), and the filled ones had the effective electrical conductivity $\sigma_{p} \gg \sigma_{m}$. The electrical conductivity of the formed mesh of resistors is calculated using the Frank-Lobb algorithm [32]. The coefficient of optical transmission $T_{r}$ was calculated as the ratio of the number of blank (transparent) cells to the total number of cells in the mesh $(m \times m)$. A more detailed description of the algorithms of calculations is given in works $[19,20]$. All calculations in this paper were performed, by using the following parameter values: $L=32 l, m=128-1024$, and $\sigma_{p} / \sigma_{m}=10^{6}$. In calculations, the results were averaged over at least 10 different $\mathrm{MC}$ experiments.

\section{Results and Discussion}

Figure 2, a shows the dependences of the electrical conductivity $\sigma$ and the optical transmittance $T_{r}$ on the numerical concentration of needles $n$ at the initial time moment, i.e., immediately after the formation of the system with the use of the RSA deposition mechanism.

As we see, an increase in $\sigma$ and a decrease in the optical transmission of $T_{r}$ are observed, as $n$ increases. At certain threshold values $n_{p}$, there is a significant increase in the electrical conductivity, which corresponds to the percolation transition of the system to a conducting state. This transition corresponds to the achievement of the mean geometric value of the con- ductivity $\left(\lg \sigma_{g}=3\right)$ and depends on $m$. For example, $n_{p}=2.3$ for $m=256(a=8)$ and $n_{p}=3.98$ for $m=512(a=16)$.

Fig. 2, $b$ shows the dependences of the electrical conductivity $\sigma$ on the optical transmission $T_{r}$ for different values of $m$. As we can see, percolation transitions are observed on these dependences for a certain critical value of the optical transmission. The insert in Fig. 2, $b$ shows the dependence of the value of $T_{r}$ for the mean geometric value of the conductivity $\left(\lg \sigma_{g}=3\right)$. This value increases with $m$ or the effective aspect ratio $a$. This indicates the possibility to obtain conductive films with high transparency, by using needles with a large aspect ratio.

Typical examples of the needle distribution pattern in the aging process for $t=10^{3}$ and $t=10^{6}$ are presented in Fig. 3, $a$ for the fixed values of the density $n=3$, energy $u_{o}=-1$, and effective radius $R_{c}=0.5,1.0,2.0$. In the process of aging, changes in the morphology depend significantly on the values of $R_{c}$. In particular, for relatively short-range potentials $\left(R_{c}=0.5,1.0\right)$ as a result of aging, island structures are formed which include the aggregates of parallel-oriented particles. For more long-range potentials $\left(R_{c}=2.0\right)$, the formation of vortex-like structures is observed at the intermediate aging stage $\left(t=10^{3}\right)$. In the long time $\left(t=10^{6}\right)$, the net-like structures are finally formed, which unite stack ag-

ISSN 2071-0194. Ukr. J. Phys. 2019. Vol. 64, No. 4 

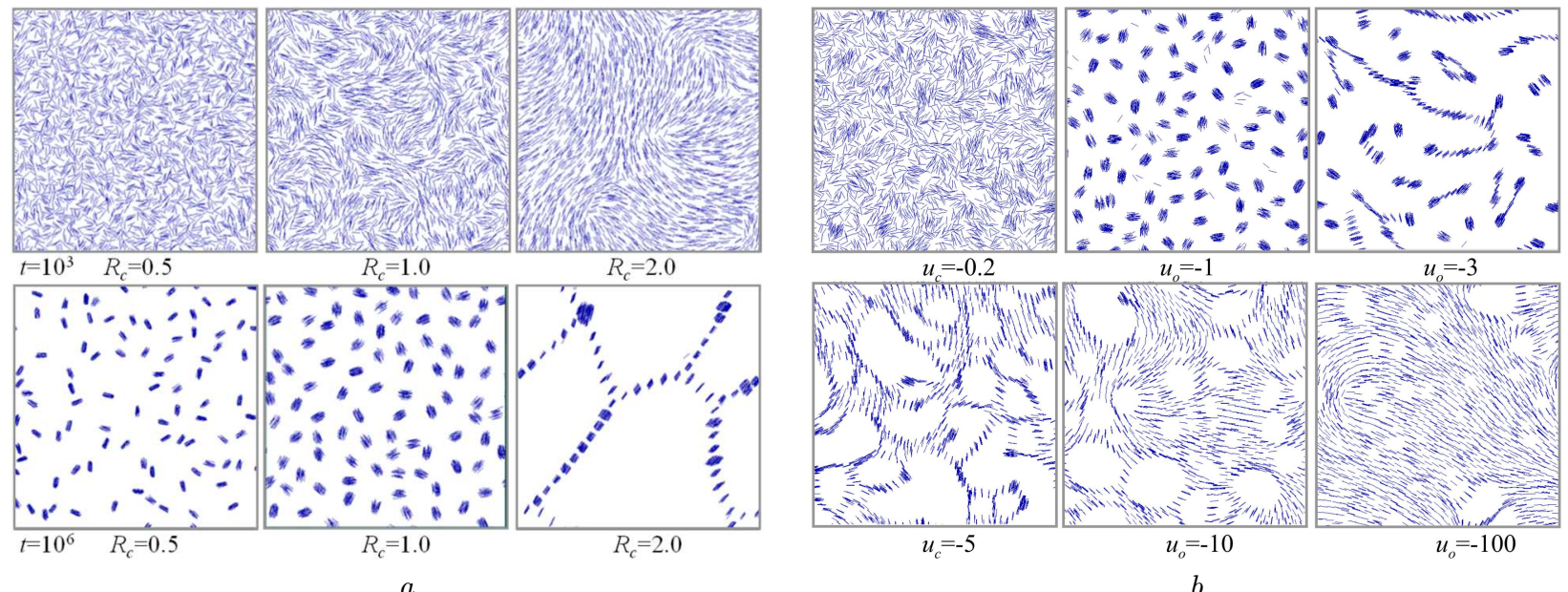

$b$

Fig. 3. Characteristic patterns of the needle distribution during aging: for $t=10^{3}$ and $t=10^{6}$ with fixed values of the density $n=3$, energy $u_{o}=-1$ (attraction) and three values of the effective radius of interaction $R_{c}=0.5,1.0,2.0 a$ ); for $t=2 \cdot 10^{4}$ with fixed values of $n=3, R_{c}=1.0$ and several values of $u_{o} b$ )
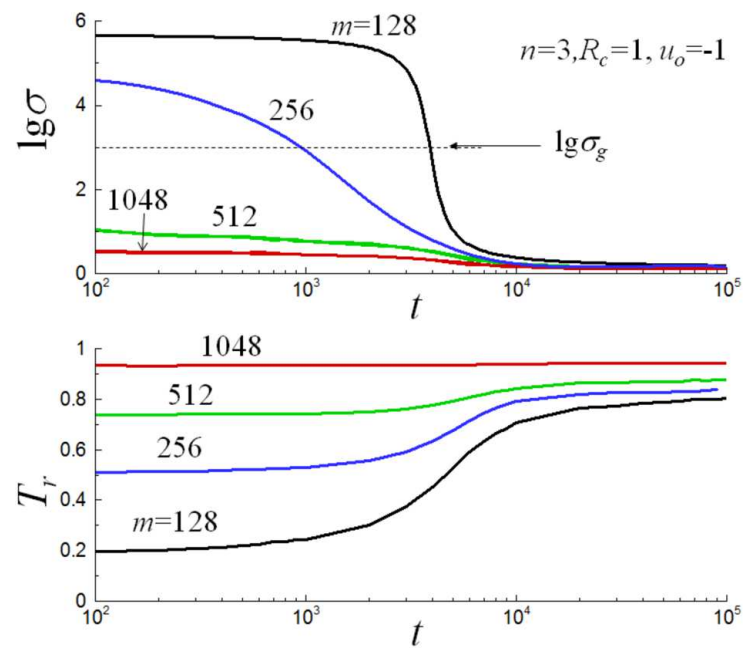

Fig. 4. Time dependences of the electrical conductivity $\sigma$ and the optical transmission $T_{r}$ for the numerical density $n=3$, interaction radius $R_{c}=1.0$, and energy $u_{o}=-1$ for different values of $m$

gregates. Thus, the amplification of the long-range interactions between particles leads to the emergence of a larger scale of the self-organization of particles.

Typical examples of the needle distribution pattern in the aging process for $t=2 \cdot 10^{4}$ are presented in Fig. $3, b$ for fixed values of the density $n=3$, effective radius $R_{c}=1.0$, and several energy values $u_{o}$. For a short-range potential $\left(R_{c}=1.0\right)$, island structures that include the aggregates of parallel-oriented particles, as a result of the aging,are formed only for relISSN 2071-0194. Ukr. J. Phys. 2019. Vol. 64, No. 4

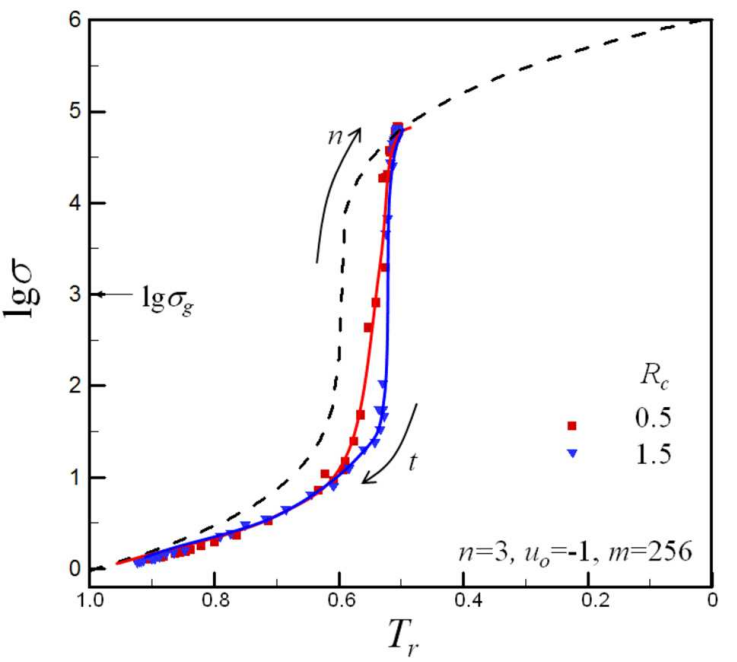

Fig. 5. Dependence of the electrical conductivity $\sigma$ on the optical transmission $T_{r}$ in the aging process at $m=256$, for the numerical concentration $n=3$, the energy $u_{o}=-1$, and the various values of the interaction radius $R_{c}=0.5$ and 1.5 . The dashed line corresponds to the initial RSA state at different values of $n$

atively small values of $\left|u_{o}\right|=1 \div 2$. For small values of $\left|u_{o}\right|=0.2$, the segregation is not observed practically. For large values of $u_{o}\left(\left|u_{o}\right| \geq 3\right)$, the formation of connected structures with significant cavities was observed.

Examples of the time dependences of the electrical conductivity $\sigma$ and the optical transmittance $T_{r}$ for $n=3, R_{c}=1.0, u_{o}=-1$, and various values of $m$ 
are shown in Fig. 4. This case corresponds to the aging process with the formation of island structures, which include the aggregates of parallel-oriented particles (Fig. 3, a). The conductivity $\sigma$ decreases over time, and the optical transmission coefficient $T_{r}$, on the contrary, increases. The processes of aging lead to the destruction of the percolation cluster. Thus, the illumination of the film occurs. Similar dependences were observed for other values of the parameters $n$, $R_{c}$, and $u_{o}$.

In Fig. 5, the electrical conductivity dependences $\sigma$ are compared with the optical transmission $T_{r}$ at $m=256$ for the state at the initial time moment (dotted line) and the time evolution in the aging process at $n=3, u_{o}=-1$, and $R_{c}=0.5$ and 1.5. The obtained $\sigma\left(T_{r}\right)$ dependences for other parameter values were quite similar to the dependence that was obtained for the state at the initial time moment. The critical optical transmittance value corresponding to the achievement of the average geometric value of the electrical conductivity $\left(\lg \sigma_{g}=3\right)$ decreases, as $R_{c}$ and $\left|u_{o}\right|$ increase. This corresponds to an increase in the connectivity of structures, while forming the mesh or cavity structures shown in Fig. 3.

\section{Conclusions}

Using the Monte-Carlo method, the changes in the electrical conductivity and optical transmission of the two-dimensional films filled with needle-like particles have been studied. The processes of aging of the system as a result of the Brownian motion of needles in the presence of an anisotropic interaction between particles are analyzed. It is shown that the anisotropic interaction can lead to a significant change in the structure, electrical conductivity, and optical transmission of films. Depending on the energy $u_{o}$ and the interaction radius $R_{c}$, the formation of island, net-like, and hole-like (with significant cavities) structures is observed. It is shown that the connectivity of structures increases with $\left|u_{o}\right|$ and $R_{c}$. These results allow us to predict the conditions of obtaining the conductive films with high optical transmission.

The authors thank Prof. Y.Y. Tarasevich for the useful advices and the assistance in performing the calculations. The work was partially supported by the State organization "Target Department of Taras Shevchenko National University of Kyiv at the $\mathrm{Na}$ - tional Academy of Sciences of Ukraine," project No. $15 F$, and project No. 43/18-N of fundamental research programs of the NAS of Ukraine "Fundamental problems of creation of new nanomaterials and nanotechnologies".

1. D.J. Lipomi, M. Vosgueritchian, B.C.K. Tee, S.L. Hellstrom, J.A. Lee, C.H. Fox, Z. Bao. Skin-like pressure and strain sensors based on transparent elastic films of carbon nanotubes. Nat. Nanotechnol. 6, 788 (2011).

2. L. Hu, D.S. Hecht, G. Grŭner. Percolation in transparent and conducting carbon nanotube networks. Nano Lett. 4, $2513(2004)$.

3. K.-Y. Chun, Y. Oh, J. Rho, J.-H. Ahn, Y.-J. Kim, H.R. Choi, S. Baik. Highly conductive, printable and stretchable composite films of carbon nanotubes and silver. Nat. Nanotechnol. 5,853 (2010).

4. D.S. Hecht, L. Hu, G. Irvin. Emerging transparent electrodes based on thin films of carbon nanotubes, graphene, and metallic nanostructures. Adv. Mater. 23, 1482 (2011).

5. Y. Leterrier, L. Medico, F. Demarco, J.-A. Månson, U. Betz, M.F. Escola, M.K. Olsson, F. Atamny. Mechanical integrity of transparent conductive oxide films for flexible polymer-based displays. Thin Solid Films 460, 156 (2004).

6. J.S. Moon, J.H. Park, T.Y. Lee, Y.W. Kim, J.B. Yoo, C.Y. Park, J.M. Kim, K.W. Jin. Transparent conductive film based on carbon nanotubes and PEDOT composites. Diam. Relat. Mater. 14, 1882 (2005).

7. M.-J. Yim, K.-W. Paik. Design and understanding of anisotropic conductive films (ACFs) for LCD packaging. In: Proceedings of the First IEEE International Symposium on Polymeric Electronics Packaging (IEEE, 1997), p. 233.

8. X. Li, Y. Zhu, W. Cai, M. Borysiak, B. Han, D. Chen, R.D. Piner, L. Colombo, R.S. Ruoff. Transfer of large-area graphene films for high-performance transparent conductive electrodes. Nano Lett. 9, 4359 (2009).

9. X. Wang, L. Zhi, K. Mŭllen. Transparent, conductive graphene electrodes for dye-sensitized solar cells. Nano Lett. 8, 323 (2008).

10. L. Lisetski, M. Soskin, N. Lebovka. Carbon nanotubes in liquid crystals: Fundamental properties and applications, in: Phys. Liq. Matter Mod. Probl. (Springer, 2015).

11. L. Jiang, L. Gao, J. Sun. Production of aqueous colloidal dispersions of carbon nanotubes. J. Colloid Interface Sci. 260, 89 (2003).

12. S.D. Bergin, V. Nicolosi, H. Cathcart, M. Lotya, D. Rickard, Z. Sun, W.J. Blau, J.N. Coleman. Large populations of individual nanotubes in surfactant-based dispersions without the need for ultracentrifugation. J. Phys. Chem. C 112, 972 (2008).

13. M. Loginov, N. Lebovka, E. Vorobiev. Laponite assisted dispersion of carbon nanotubes in water. J. Colloid Interface Sci. 365, 127 (2012).

ISSN 2071-0194. Ukr. J. Phys. 2019. Vol. 64, No. 4 
14. V. Tohver, J.E. Smay, A. Braem, P. V Braun, J.A. Lewis. Nanoparticle halos: A new colloid stabilization mechanism. Proc. Natl. Acad. Sci. 98, 8950 (2001).

15. O. Yaroshchuk, S. Tomylko, O. Kovalchuk, N. Lebovka. Liquid crystal suspensions of carbon nanotubes assisted by organically modified Laponite nanoplatelets. Carbon 68, 389 (2014).

16. B. Smith, K. Wepasnick, K.E. Schrote, H.-H. Cho, W.P. Ball, D.H. Fairbrother. Influence of surface oxides on the colloidal stability of multi-walled carbon nanotubes: A structure-property relationship. Langmuir 25, 9767 (2009).

17. M. Farbod, S.K. Tadavani, A. Kiasat. Surface oxidation and effect of electric field on dispersion and colloids stability of multiwalled carbon nanotubes. Colloids Surfaces A 384, 685 (2011).

18. Y.Y. Tarasevich, V.V. Laptev, V.A. Goltseva, N.I. Lebovka. Influence of defects on the effective electrical conductivity of a monolayer produced by random sequential adsorption of linear $k$-mers onto a square lattice. Phys. A. Stat. Mech. Its Appl. 477, 195 (2017).

19. Y.Y. Tarasevich, N.I. Lebovka, I.V. Vodolazskaya, A.V. Eserkepov, V.A. Goltseva, V.V. Chirkova. Anisotropy in electrical conductivity of two-dimensional films containing aligned nonintersecting rodlike particles: Continuous and lattice. Phys. Rev. E 98, 12105 (2018).

20. N.I. Lebovka, Y.Y. Tarasevich, N.V. Vygornitskii, A.V. Eserkepov, R.K. Akhunzhanov. Anisotropy in electrical conductivity of films of aligned intersecting conducting rods. Phys. Rev. E 98, 12104 (2018).

21. Y.Y. Tarasevich, I.V. Vodolazskaya, A.V. Eserkepov, V.A. Goltseva, P.G. Selin, N.I. Lebovka. Simulation of the electrical conductivity of two-dimensional films with aligned rod-like conductive fillers: Effect of the filler length dispersity. J. Appl. Phys. 124, 145106 (2018).

22. N.I. Lebovka, Y.Y. Tarasevich, V.A. Gigiberiya, N.V. Vygornitskii, Diffusion-driven self-assembly of rod-like particles: Monte-Carlo simulation on a square lattice. Phys. Rev. E 95, 52130 (2017).

23. Y.Y. Tarasevich, V.V. Laptev, A.S. Burmistrov, N.I. Lebovka. Pattern formation in a two-dimensional two-species diffusion model with anisotropic nonlinear diffusivities: a lattice approach. J. Stat. Mech. Theory Exp. 2017, 093203 (2017).

24. Y.Y. Tarasevich, V.V. Laptev, A.S. Burmistrov, N.I. Lebovka. Effect of aging on electrical conductivity of two- dimensional composite with rod-like fillers. J. Phys. Conf. Ser. 955, 12006 (2018).

25. N.I. Lebovka, Y.Y. Tarasevich, N.V. Vygornitskii. Vertical drying of a suspension of sticks: Monte Carlo simulation for continuous two-dimensional problem. Phys. Rev. E 97, 22136 (2018).

26. N.I. Lebovka, N.V. Vygornitskii, L.A. Bulavin, L.O. Mazur, L.N. Lisetski, Monte Carlo studies of optical transmission of anisotropic suspensions, J. Mol. Liq. 272, 1025 (2018).

27. J.W. Evans. Random and cooperative sequential adsorption. Rev. Mod. Phys. 65, 1281 (1993).

28. G. Li, J.X. Tang. Diffusion of actin filaments within a thin layer between two walls. Phys. Rev. E 69, 61921 (2004).

29. S. Broersma. Rotational diffusion constant of a cylindrical particle. J. Chem. Phys. 32, 1626 (1960).

30. S. Broersma. Viscous force constant for a closed cylinder. J. Chem. Phys. 32, 1632 (1960).

31. S. Broersma. Viscous force and torque constants for a cylinder. J. Chem. Phys. 74, 6989 (1981).

32. D.J. Frank, C.J. Lobb. Highly efficient algorithm for percolative transport studies in two dimensions. Phys. Rev. B 37, 302 (1988).

Received 06.02.19

Л.О. Мазур, Л.А.Булавін,

М.В. Вигорницъкий, М.І. Лебовка

ВПЛИВ БРОУНІВСЬКОГО РУХУ

НА ЕЛЕКТРОПРОВІДНІСТЬ ТА ПРОЗОРІСТЬ

ДВОВИМІРНИХ ПЛІВОК, НАПОВНЕНИХ

ГОЛКОПОДІБНИМИ ЧАСТИНКАМИ

$\mathrm{P}$ е $з$ ю м е

За допомогою методу Монте-Карло (MC) досліджено вплив броунівського руху на електропровідність та оптичну прозорість двовимірних плівок, наповнених голкоподібними частинками (голками). Початковий стан системи одержували при використанні процесу випадкової послідовної адсорбції (RSA). При подальшій еволюції (старінні) системи були враховані трансляційні і обертальні дифузійні рухи. Перетин між голками було заборонено. Потенціал взаємодії між голками був короткосяжним (був ненульовим при відстані між голками менше, ніж $R_{c}$ ) і залежав від кута між голками $\phi\left(\propto \cos ^{2} \phi\right)$. В залежності від параметрів потенціалу взаємодії старіння призводило до утворення острівкових, сіткоподібних та діркоподібних структур. Аналізується залежність між електропровідністю та оптичною прозорістю при старінні. 